Preprint

CHIBA-EP-105

hep-th/9803133

(revised)

June 1998

\title{
Existence of Confinement Phase in Quantum Electrodynamics
}

\author{
Kei-Ichi Kondo ${ }^{1 \dagger}$ \\ ${ }^{1}$ Department of Physics, Faculty of Science, Chiba University, Chiba 263, Japan \\ † E-mail: kondo@cuphd.nd.chiba-u.ac.jp
}

\begin{abstract}
We show that the four-dimensional U(1) gauge theory in the continuum formulation has a confining phase (exhibiting area law of the Wilson loop) in the strong coupling region above a critical coupling $g_{c}$. This result is obtained by taking into account topological non-trivial sectors in $\mathrm{U}(1)$ gauge theory. The derivation is based on the reformulation of gauge theory as a deformation of a topological quantum field theory and subsequent dimensional reduction of the D-dimensional topological quantum field theory to the (D-2)-dimensional nonlinear sigma model. The topological quantum field theory part of the four-dimensional $\mathrm{U}(1)$ gauge theory is exactly equivalent to the two-dimensional $\mathrm{O}(2)$ nonlinear sigma model. The confining (resp. Coulomb) phase of $\mathrm{U}(1)$ gauge theory corresponds to the high (resp. low) temperature phase of $\mathrm{O}(2)$ nonlinear sigma model and the critical point $g_{c}$ is determined by the BerezinskiiKosterlitz-Thouless phase transition temperature. The quark (charge) confinement in the strong coupling phase is caused by the vortex condensation. Thus the continuum gauge theory has the direct correspondence to the compact formulation of lattice gauge theory.
\end{abstract}

Key words: quark confinement, abelian gauge theory, topological quantum field theory, dimensional reduction, nonlinear sigma model, instanton, vortex, monopole

PACS: 12.38.Aw, 12.38.Lg

* To be published in Phys. Rev. D. 


\section{Introduction}

In this paper we study the phase structure of the continuum Abelian U(1) gauge theory by including the effect due to compactness of $\mathrm{U}(1)$ group. The reason of taking into compactness is as follows. From the viewpoint of unified field theory, the Abelian group should be embedded as a subgroup in larger non-Abelian gauge group. In view of this, the Abelian group should be compact. Another importance of compactness of Abelian gauge group stems from a possibility of explaining quantization of charge [1]. In non-compact QED there are no reasons for charge quantization.

In this paper we show that the four-dimensional $\mathrm{U}(1)$ gauge theory has a confinement phase in the strong coupling region $g>g_{c}$ due to the compactness (periodicity) leading to the non-trivial topological configuration. If we neglect the periodicity, we have a free $\mathrm{U}(1)$ gauge theory which has only one phase, the Coulomb phase, as expected. This work confirms the claim made by Polyakov [2, 3]. However, the claim that the abelian gauge theory has a confinement phase sounds strange from the conventional wisdom based on the continuum abelian gauge theory. We clarify the meaning of this statement in what follows.

More than twenty years ago, it has been pointed out by many authors that the four-dimensional SU(2) non-Abelian gauge theory bears many similarities with a twodimensional $\mathrm{O}(3)$ nonlinear sigma model (NLSM). Both theories possess asymptotic freedom, multi-instanton (and anti-instanton) solution, dynamical mass generation and scale invariance (i.e., no intrinsic scale parameter), see [4] for references.

These similarities can be seen also in the lattice regularized versions of these models, between spin models and lattice gauge theories [5]. Naively the scaling limit of the classical $\mathrm{O}(3)$ Heisenberg model is the $\mathrm{O}(3)$ NLSM, whereas that of the $\mathrm{SU}(2)$ lattice gauge theory is the $\mathrm{SU}(2)$ gauge theory. One can take the scaling limit of a lattice theory at a second order phase transition point. Hence the scaling limit is taken by approaching the critical point $T \rightarrow T_{c}$ (or $g \rightarrow g_{c}$ ) as the lattice spacing $a$ goes to zero, $a \rightarrow 0$, in such a way that the physical quantities remain finite.

In two-dimensional classical $\mathrm{O}(3)$ Heisenberg model, the two-point correlation function decays exponentially at any finite temperature. This corresponds to a claim in four-dimensional lattice $\mathrm{SU}(2)$ gauge theory that confinement phase survives as long as the coupling constant $g$ is positive, even if $g \ll 1$. Both models have a phase transition at $T=0(g=0)$, i.e., $T_{c}=0\left(g_{c}=0\right)$ which is believed to be the second order.

In a previous paper [4], it has been shown that these similarities between two models are not merely an accident; actually we have proved the exact equivalence between (D-2)-dimensional O(3) NLSM and the $D$-dimensional topological quantum field theory (TQFT) obtained by removing the perturbative deformation (topological trivial sector) from the $D$-dimensional $\mathrm{SU}(2)$ non-Abelian gauge theory $(D \geq 3)$. This proof is based on an idea of the dimensional reduction of Parisi and Sourlas [6]. The case of $D=4$ is the most interesting case of physical reality.

What can we say in the Abelian case? For this, recall a fact that the twodimensional $\mathrm{O}(2)$ NLSM or XY model undergoes a phase transition without the appearance of a spontaneous magnetization. This absence of order parameter in two 
dimensions is consistent with the Coleman-Mermin-Wagner (CMW) theorem [7]. The low temperature phase $\left(T<T_{c}\right)$ contains massless spin waves. On the other hand, the high temperature phase $\left(T>T_{c}\right)$ is completely disordered. For this phase transition, the periodicity of the angular variable $\varphi$ is quite essential. The model has topological singularities, called the vortices. These vortices condense in high temperature and disorder the correlation function [8]. This phase transition is called the BerezinskiiKosterlitz-Thouless (BKT) transition [8]. The vortex part is equivalent to the neutral Coulomb gas and sine-Gordon theory, see [9, 10, 11]. Although the existence of BKT transition is rather subtle, the existence of BKT transition was rigorously proved by Fröhlich and Spencer [12].

In lattice formulation, it is well known that all of these properties in two-dimensional abelian spin models have correspondences in the Abelian gauge theory in four dimensions. The vortices in two dimensions are closely related to magnetic monopoles in four dimensions, see [13, 14, 15]. The condensation of closed loops of magnetic monopole currents leads to quark (charge) confinement in the strongly coupled phase of Abelian gauge theory, since electric flux cannot easily penetrate such a medium (which we call the dual Meissner effect). It will be worth remarking that the dual superconductor vacuum of quantum chromodynamics (QCD) has been derived recently without any ad hoc assumption [16] from QCD in the continuum formulation.

In lattice gauge theory, charge confinement in the sense of area decay of the Wilson loop is derived in the strong coupling region by using the strong coupling expansion [5, 17]. Quite remarkably, the quark (charge) confinement in lattice gauge theory occurs irrespective of the details of the gauge group, as long as it is compact (discrete [18, 19] or continuous), even for abelian gauge group. However, one expects that the $\mathrm{U}(1)$ lattice gauge theory in four dimensions $\left(U(1)_{4}\right)$ has a Coulomb phase in the weak coupling region, which was proved rigorously by Guth 20] and Fröhlich and Spencer [21]. Therefore the U(1) lattice gauge theory undergoes a phase transition at a finite non-zero coupling $g_{c}$. In continuum gauge theory, such non-trivial phase structure was suggested to occur due to topological non-trivial configurations by Polyakov [2, 1]. Actually he has shown the confinement phase in three-dimensional $\mathrm{U}(1)$ gauge theory for arbitrary gauge coupling, in agreement with the lattice analysis. In four dimensions, he claimed that the weak coupling $\mathrm{U}(1)$ gauge theory does not confine. Accordingly, it is expected that the U(1) gauge theory in four dimensions have two phases, confinement and deconfinement (Coulomb) phases, whereas only one phase i.e., confinement phase exists in three dimensions.

In this paper, we show that the continuum four-dimensional U(1) gauge theory has two phases, (strong coupling) confinement phase and (weak coupling) Coulomb phase, which have direct correspondence with the high-temperature and low-temperature phases in $\mathrm{O}(2)$ NLSM respectively. The phase transition point corresponds to the BKT transition in XY model. Therefore, the phase transition point $g_{c}$ is determined by the BKT transition temperature $T_{c}$. This is one of the main results of this paper. This result is obtained as a specific case of the previous paper [四]

Therefore, in the strong coupling phase $\left(g>g_{c}\right)$, the continuum $\mathrm{U}(1)$ gauge theory confines quarks and the gauge field becomes massive, in agreement with the result of lattice gauge theory. In the weak coupling phase, on the other hand, quarks are 
liberated and gauge field remains massless. In the weak coupling phase $\left(g<g_{c}\right)$, the beta function of the renormalization group [22] is identically zero and $0<g<g_{c}$ is the line of fixed points, if we neglect the perturbative deformations. For a review of lattice gauge theory, see e.g. 23, 24, 25].

The plan of this paper is as follows. In section 2, we give the reformulation of Abelian gauge theory as a deformation of a topological quantum field theory. In section 3, using the reformulation of section 2, we evaluate the Wilson loop expectation value in four-dimensional Abelian gauge theory. In the final section we discuss the renormalization group properties and extension of our scheme to other dimensional cases. More details about the interplay between the Abelian and non-Abelian cases are given in a forthcoming paper.

\section{Abelian gauge theory as a deformation of TQFT and dimensional reduction}

Now we reformulate quantum electrodynamics (QED) as a deformation of a topological quantum field theory. It is obtained as a special case of non-Abelian gauge theory given in the previous paper [4].

\subsection{Decomposition into perturbative and topological non- trivial sectors}

QED on the $D$-dimensional space-time is defined by the action,

$$
\begin{aligned}
S_{Q E D}^{t o t} & =\int d^{D} x\left(\mathcal{L}_{Q E D}\left[a_{\mu}, \psi\right]+\mathcal{L}_{G F}\right), \\
\mathcal{L}_{Q E D}\left[a_{\mu}, \psi\right] & :=-\frac{1}{4} f_{\mu \nu} f_{\mu \nu}+\bar{\psi}\left(i \gamma^{\mu} D_{\mu}[a]-m\right) \psi,
\end{aligned}
$$

where

$$
\begin{aligned}
f_{\mu \nu}(x) & :=\partial_{\mu} a_{\nu}(x)-\partial_{\nu} a_{\mu}(x), \\
D_{\mu}[a] & :=\partial_{\mu}-i g a_{\mu} .
\end{aligned}
$$

The gauge transformation of the $\mathrm{U}(1)$ gauge field $a_{\mu}(x)$ and the fermion field $\psi$ is defined by

$$
\begin{aligned}
a_{\mu}(x) & \rightarrow a_{\mu}^{U}(x):=a_{\mu}(x)+\frac{i}{g} U(x) \partial_{\mu} U^{\dagger}(x), \quad U(x) \in U(1) \\
\psi(x) & \rightarrow \psi^{U}(x):=U(x) \psi(x) .
\end{aligned}
$$

The gauge fixing term $\mathcal{L}_{G F}$ is given by

$$
\mathcal{L}_{G F}:=-i \delta_{B} G_{g f}\left[a_{\mu}, C, \bar{C}, \phi\right],
$$


using the nilpotent Becchi-Rouet-Stora-Tyupin (BRST) transformation $\delta_{B}$,

$$
\begin{aligned}
\delta_{B} a_{\mu}(x) & =\partial_{\mu} C(x), \\
\delta_{B} C(x) & =0, \\
\delta_{B} \bar{C}(x) & =i \phi(x), \\
\delta_{B} \phi(x) & =0, \\
\delta_{B} \psi(x) & =i g C(x) \psi(x), \quad \delta_{B} \bar{\psi}(x)=-i g C(x) \bar{\psi}(x),
\end{aligned}
$$

where $\phi$ is the Lagrange multiplier field.

The partition function of QED with the source term

$$
S_{J}\left[a_{\mu}, C, \bar{C}, \phi, \psi, \bar{\psi}\right]:=\int d^{D} x\left(J^{\mu} a_{\mu}+J_{c} C+J_{\bar{c}} \bar{C}+J_{\phi} \phi+\bar{\eta} \psi+\eta \bar{\psi}\right)
$$

is given by

$$
Z_{Q E D}[J]:=\int\left[d a_{\mu}\right][d C][d \bar{C}][d \phi][d \psi][d \bar{\psi}] \exp \left\{i S_{Q E D}^{t o t}+i S_{J}\right\} .
$$

To reformulate QED as a deformation of a topological quantum field theory according to $\mathbb{4}$, we first regard the $\mathrm{U}(1)$ gauge field $a_{\mu}$ and the fermion field $\psi$ as the gauge transformation of the $\mathrm{U}(1)$ gauge fields $v_{\mu}$ and $\Psi$,

$$
\begin{aligned}
a_{\mu}(x) & :=v_{\mu}(x)+\omega_{\mu}(x), \quad \omega_{\mu}(x):=\frac{i}{g} U(x) \partial_{\mu} U^{\dagger}(x) . \\
\psi(x) & :=U(x) \Psi(x),
\end{aligned}
$$

Here $v_{\mu}$ and $\Psi$ are identified with the field variables in the perturbative (topological trivial) sector $(Q=0)$, whereas $\omega_{\mu}$ belongs to the topological nontrivial sector $(Q \neq$ $0)$.

Furthermore we introduce new ghost field $\gamma$, anti-ghost field $\bar{\gamma}$ and the Lagrange multiplier field $\beta$ in the perturbative sector. They are subject to a new BRST transformation $\tilde{\delta}_{B}$,

$$
\begin{aligned}
\tilde{\delta}_{B} v_{\mu}(x) & =\partial_{\mu} \gamma(x), \\
\tilde{\delta}_{B} \gamma(x) & =0, \\
\tilde{\delta}_{B} \bar{\gamma}(x) & =i \beta(x), \\
\tilde{\delta}_{B} \beta(x) & =0, \\
\tilde{\delta}_{B} \Psi(x) & =i g \gamma(x) \Psi(x), \quad \tilde{\delta}_{B} \bar{\Psi}(x)=-i g \gamma(x) \bar{\Psi}(x),
\end{aligned}
$$

Then the partition function of QED is rewritten as

$$
\begin{aligned}
Z_{Q E D}[J]= & \int[d U][d C][d \bar{C}][d \phi] \int\left[d v_{\mu}\right][d \gamma][d \bar{\gamma}][d \beta][d \Psi][d \bar{\Psi}] \\
& \times \exp \left\{i \int d^{D} x\left(-i \delta_{B} G_{g f}\left[\omega_{\mu}+v_{\mu}, C, \bar{C}, \phi\right]\right)\right. \\
& +i \int d^{D} x\left(\mathcal{L}_{Q E D}[v, \Psi]-i \tilde{\delta}_{B} \tilde{G}_{g f}\left[v_{\mu}, \gamma, \bar{\gamma}, \beta\right]\right) \\
& \left.+i S_{J}\left[\omega_{\mu}+v_{\mu}, C, \bar{C}, \phi, U \Psi, \bar{\Psi} U^{\dagger}\right]\right\}
\end{aligned}
$$

\footnotetext{
1 The decomposition of $a_{\mu}, a_{\mu}=v_{\mu}+\omega_{\mu}$, corresponds to the superposition of two independent configuration, $\varphi=\varphi_{S W}+\varphi_{V}$ (spin waves and vortex parts) in XY model.
} 
where $\tilde{G}_{g f}$ is a gauge fixing functional for the perturbative (topological trivial) sector.

\subsection{Gauge fixing}

The Lorentz gauge is given by

$$
F[a]:=\partial_{\mu} a^{\mu}=0 .
$$

The most familiar choice of $G_{g f}$

$$
G_{g f}=\bar{C}\left(\partial_{\mu} a^{\mu}+\frac{\alpha}{2} \phi\right)
$$

yields the familiar form of the gauge fixing term,

$$
\mathcal{L}_{G F}:=-i \delta_{B} G_{g f}\left[a_{\mu}, C, \bar{C}, \phi\right]=\phi \partial_{\mu} a^{\mu}+i \bar{C} \partial^{\mu} \partial_{\mu} C+\frac{\alpha}{2} \phi^{2} .
$$

In this paper we propose to use a choice,

$$
G_{g f}^{U(1)}=-\bar{\delta}_{B}\left(\frac{1}{2} a_{\mu}^{2}+i C \bar{C}\right)
$$

where $\bar{\delta}_{B}$ is the anti-BRST transformation [1],

$$
\begin{aligned}
\bar{\delta}_{B} a_{\mu}(x) & =\partial_{\mu} \bar{C}(x), \\
\bar{\delta}_{B} C(x) & =i \bar{\phi}(x), \\
\bar{\delta}_{B} \bar{C}(x) & =0, \\
\bar{\delta}_{B} \bar{\phi}(x) & =0, \\
\bar{\delta}_{B} \psi(x) & =\bar{C}(x) \psi(x), \\
\phi(x)+\bar{\phi}(x) & =0,
\end{aligned}
$$

where $\bar{\phi}$ is defined in the last equation.

Apart from a total derivative term, this choice yields

$$
\mathcal{L}_{G F}=i \delta_{B} \bar{\delta}_{B}\left(\frac{1}{2} a_{\mu}^{2}+i C \bar{C}\right)=-i \delta_{B}\left[\bar{C}\left(\partial_{\mu} a^{\mu}-\phi\right)\right]
$$

Therefore, the choice (2.18) corresponds in (2.17) to the choice of the gauge-fixing parameter,

$$
\alpha=-2
$$

which has appeared also in the non-Abelian case [4]. The above choice for $G_{g f}^{U(1)}$ yields the decomposition,

$$
\begin{aligned}
\mathcal{L}_{G F} & =-i \delta_{B} G_{g f}^{U(1)}\left[\omega_{\mu}+v_{\mu}, C, \bar{C}, \phi\right] \\
& =i \delta_{B} \bar{\delta}_{B}\left(\frac{1}{2}\left(\omega_{\mu}+v_{\mu}\right)^{2}+i C \bar{C}\right) \\
& =\mathcal{L}_{T Q F T}+i v_{\mu} \delta_{B} \bar{\delta}_{B} \omega_{\mu},
\end{aligned}
$$


where we have defined

$$
\mathcal{L}_{T Q F T}:=i \delta_{B} \bar{\delta}_{B}\left(\frac{1}{2} \omega_{\mu}^{2}+i C \bar{C}\right)
$$

Here we have used that the action of $\delta_{B}$ is trivial in the perturbative sector,

$$
\delta_{B} v_{\mu}=0=\bar{\delta}_{B} v_{\mu}
$$

while

$$
\delta_{B} \omega_{\mu}=\partial_{\mu} C, \quad \bar{\delta}_{B} \omega_{\mu}=\partial_{\mu} \bar{C}
$$

\subsection{Deformation of topological quantum field theory}

Finally, the partition function of QED is cast into the form,

$$
\begin{aligned}
Z_{Q E D}[J]:= & \int[d U][d C][d \bar{C}][d \phi] \exp \left\{i S_{T Q F T}\left[\omega_{\mu}, C, \bar{C}, \phi\right]\right. \\
& \left.+i \int d^{D} x\left[J^{\mu} \omega_{\mu}+J_{c} C+J_{\bar{c}} \bar{C}+J_{\phi} \phi\right]+i W\left[U ; J^{\mu}, \bar{\eta}, \eta\right]\right\},
\end{aligned}
$$

where $W\left[U ; J^{\mu}, \bar{\eta}, \eta\right]$ is the generating functional of QED in the perturbative sector (pQED) given by

$$
\begin{aligned}
e^{i W\left[U ; J^{\mu}, \bar{\eta}, \eta\right]}:= & \int\left[d v_{\mu}\right][d \gamma][d \bar{\gamma}][d \beta][d \Psi][d \bar{\Psi}] \exp \left\{i S_{p Q E D}[v, \Psi, \gamma, \bar{\gamma}, \beta]\right. \\
& \left.+i \int d^{D} x\left[v_{\mu} \mathcal{J}_{\mu}+\bar{\eta} U \Psi+\eta \bar{\Psi} U^{\dagger}\right]\right\} \\
S_{p Q E D}[v, \Psi, \gamma, \bar{\gamma}, \beta]:= & \int d^{D} x\left[\mathcal{L}_{Q E D}[v, \Psi]-i \tilde{\delta}_{B} \tilde{G}_{g f}\left(v_{\mu}, \gamma, \bar{\gamma}, \beta\right)\right] \\
\mathcal{J}_{\mu}:= & J_{\mu}+i \delta_{B} \bar{\delta}_{B} \omega_{\mu} .
\end{aligned}
$$

The correlation functions of the original (fundamental) field $a_{\mu}, \psi, \bar{\psi}$ are obtained by differentiating $Z_{Q E D}[J]$ with respect to the corresponding source $J_{\mu}, \bar{\eta}, \eta$.

All the field configurations are classified according to the integer-valued topological charge $Q$ which is specified later. The above reformulation of gauge theory is the decomposition of the original theory into the topological trivial sector with $Q=0$ and topological non-trivial sector with $Q \neq 0$. This corresponds to the decomposition of XY model into spin wave part $(Q=0)$ and the vortex part $(Q \neq 0)$ where $Q$ is given by the winding number of the vortex solution. However, the XY model is not a gauge theory and does not have any local gauge invariance.

The integration over the fields $(U, C, \bar{C}, \phi)$ in TQFT should be treated nonperturbatively by taking into account the topological non-trivial configurations. The deformation $W\left[U ; J^{\mu}, \bar{\eta}, \eta\right]$ from the TQFT should be calculated according to the ordinary perturbation theory in the coupling constant $g$. The perturbative expansion around the TQFT means the integration over the new fields $\left(v_{\mu}, \gamma, \bar{\gamma}, \beta\right)$ based on the perturbative expansion in powers of the coupling constant $g$. 


\subsection{Dimensional reduction to $\mathrm{O}(2)$ NLSM}

Following the argument given in [4] based on the Parisi-Sourlas dimensional reduction, it turns out that the D-dimensional TQFT (as the topological non-trivial sector of D-dimensional $\mathrm{U}(1)$ abelian gauge theory) with an action

$$
S_{T Q F T}\left[\omega_{\mu}, C, \bar{C}, \phi\right]=\int d^{D} x i \delta_{B} \bar{\delta}_{B}\left(\frac{1}{2} \omega_{\mu}(x) \omega_{\mu}(x)+i C(x) \bar{C}(x)\right)
$$

is equivalent to the (D-2)-dimensional O(2) NLSM with an action,

$$
\begin{aligned}
S_{O(2) N L S M}[U] & :=2 \pi \int d^{D-2} z \frac{1}{2} \omega_{\mu}(z) \omega_{\mu}(z), \quad \omega_{\mu}(z):=\frac{i}{g} U(z) \partial_{\mu} U^{\dagger}(z), \\
& =\int d^{D-2} z \frac{\pi}{g^{2}} \partial_{\mu} U(z) \partial_{\mu} U^{\dagger}(z) .
\end{aligned}
$$

Dimensional reduction is due to a fact that the action (2.31) has a hidden supersymmetry and can be rewritten in the $O S p(D / 2)$ symmetric form in the superspace formulation, see 4 .

\section{Quark confinement in Abelian gauge theory}

Now we calculate the Wilson loop expectation in the U(1) gauge theory based on the reformulation given in the previous section. In what follows we move to the Euclidean formulation.

\subsection{Dimensional reduction of Wilson loop}

We define the Wilson loop operator for the closed loop $C$ by

$$
W_{C}[a]=\exp \left(i q \oint_{C} a_{\mu}(x) d x^{\mu}\right)
$$

where $q$ is a test charge. In the abelian gauge theory, the Wilson loop factorizes,

$$
W_{C}[a]=\exp \left(i q \oint_{C} \omega_{\mu}(x) d x^{\mu}\right) \exp \left(i q \oint_{C} v_{\mu}(x) d x^{\mu}\right)=: W_{C}[\omega] W_{C}[v] .
$$

For $Q=0$ sector, we choose the gauge-fixing function,

$$
\tilde{G}_{g f}=\bar{\gamma}\left(\partial_{\mu} v^{\mu}+\frac{\xi}{2} \beta\right)
$$

with a gauge-fixing parameter $\xi$. For the $\mathrm{U}(1)$ gauge theory with an action (omitting matter fields),

$$
S_{p U(1)}[v, \gamma, \bar{\gamma}, \beta]:=\frac{1}{4 g^{2}}\left(\partial_{\mu} v_{\nu}-\partial_{\mu} v_{\nu}\right)^{2}-i \tilde{\delta}_{B} \tilde{G}_{g f}
$$


the perturbative part is given by

$$
e^{i W[\omega, J, 0,0]}=\int\left[d v_{\mu}\right][d \gamma][d \bar{\gamma}][d \beta] \exp \left\{-S_{p U(1)}[v, \gamma, \bar{\gamma}, \beta]+i \int d^{D} x v_{\mu} \mathcal{J}_{\mu}\right\} .
$$

Integrating out the fields $\gamma, \bar{\gamma}, \beta$ yields

$$
e^{i W[\omega, J, 0,0]}=\int\left[d v_{\mu}\right] \exp \left\{-S[v]-\int d^{D} x^{i v_{\mu}}(x)\left[J_{\mu}(x)+i \delta_{B} \bar{\delta}_{B} \omega_{\mu}(x)\right]\right\}
$$

where

$$
S[v]:=\int d^{D} x\left[\frac{1}{4 g^{2}}\left(\partial_{\mu} v_{\nu}-\partial_{\mu} v_{\nu}\right)^{2}+\frac{1}{2 \xi}\left(\partial_{\mu} v^{\mu}\right)^{2}\right] .
$$

For the calculation of the Wilson loop expectation, $J^{\mu}$ is taken to be the current along the closed loop $C$ such that

$$
\int d^{D} x v_{\mu}(x) J^{\mu}(x)=q \oint v_{\mu}(x) d x^{\mu} .
$$

It is easy to see that

$$
e^{i W[\omega, J, 0,0]}=\left\langle W_{C}[v] e^{\left(v_{\mu}, \delta_{B} \bar{\delta}_{B} \omega_{\mu}\right)}\right\rangle_{p U(1)} \int\left[d v_{\mu}\right] e^{-S[v]+\left(v_{\mu}, \delta_{B} \bar{\delta}_{B} \omega_{\mu}\right)}
$$

where

$$
\left(v_{\mu}, \delta_{B} \bar{\delta}_{B} \omega_{\mu}\right):=\int d^{D} x v_{\mu}(x) \delta_{B} \bar{\delta}_{B} \omega_{\mu}(x) .
$$

The Wilson loop expectation is rewritten as

$$
\left\langle W_{C}[a]\right\rangle_{U(1)}=\frac{\left\langle W_{C}[\omega] e^{i W[\omega, J, 0,0]}\right\rangle_{T Q F T}}{\left\langle e^{i W[\omega, 0,0,0]}\right\rangle_{T Q F T}}
$$

where

$$
e^{i W[\omega, 0,0,0]}=\left\langle e^{\left(v_{\mu}, \delta_{B} \bar{\delta}_{B} \omega_{\mu}\right)}\right\rangle_{p U(1)} \int\left[d v_{\mu}\right] e^{-S[v]+\left(v_{\mu}, \delta_{B} \bar{\delta}_{B} \omega_{\mu}\right)}
$$

Expanding the exponential

$$
e^{\left(v_{\mu}, \delta_{B} \bar{\delta}_{B} \omega_{\mu}\right)}=e^{\int d^{D} x \delta_{B} \bar{\delta}_{B}\left(v_{\mu} \omega_{\mu}\right)(x)}=e^{\int d^{D} x\left\{Q_{B}, \bar{\delta}_{B}\left(v_{\mu} \omega_{\mu}\right)(x)\right\}}
$$

into power series and using a fact that the vacuum of TQFT obeys

$$
Q_{B}|0\rangle_{T Q F T}=0
$$

we find that this term does not contribute to the expectation value (3.11). Therefore, the Wilson loop expectation is completely separated into the topological part $(Q \neq 0)$ and the perturbative part $(Q=0)$,

$$
\left\langle W_{C}[a]\right\rangle_{U(1)}=\left\langle W_{C}[\omega]\left\langle W_{C}[v]\right\rangle_{p U(1)}\right\rangle_{T Q F T}=\left\langle W_{C}[\omega]\right\rangle_{T Q F T}\left\langle W_{C}[v]\right\rangle_{p U(1)}
$$


This corresponds to the result eq.(10) of Polyakov [2]. This property does not hold in the non-Abelian case, which makes the systematic calculation rather difficult.

In order to use the dimensional reduction for calculating the topological part, we choose the loop $C$ so that $C$ is contained in the $D-2$ dimensional space. For $D=4$, the loop $C$ must be planar. Then the dimensional reduction of the topological part leads to the equivalence of the Wilson loop expectation value between the Ddimensional U(1) TQFT and (D-2)-dimensional O(2) NLSM,

$$
\left\langle W_{C}[\omega(z)]\right\rangle_{T Q F T_{D}}=\left\langle W_{C}[\omega(z)]\right\rangle_{O(2) N L S M_{D-2}}, \quad z \in R^{D-2} .
$$

if the Wilson loop has its support on the (D-2)-dimensional space on which the NLSM is defined. Hence, the calculation of the Wilson loop in the four-dimensional U(1) gauge theory is reduced to those in the two-dimensional O(2) NLSM and the fourdimensional perturbative $\mathrm{U}(1)$ gauge theory,

$$
\left\langle W_{C}[a]\right\rangle_{U(1)_{D}}=\left\langle W_{C}[\omega]\right\rangle_{O(2) N L S M_{D-2}}\left\langle W_{C}[v]\right\rangle_{p U(1)_{D}}
$$

For large rectangular loop with sides $R, T$, the static potential is obtained by

$$
V(R)=\lim _{T \rightarrow \infty} \frac{-1}{T} \ln \left\langle W_{C}[a]\right\rangle .
$$

In four dimensions $(D=4)$, it is well known [23] that for large Wilson loop $\left\langle W_{C}[v]\right\rangle_{p U(1)_{4}}$ gives the Coulomb potential,

$$
V(R)=-\frac{g^{2}}{4 \pi} \frac{1}{R}+\text { constant }
$$

For a derivation, see e.g. Appendix of [26].

In the following, we show that $\left\langle W_{C}[\omega]\right\rangle_{O(2) N L S M_{2}}$ exhibits area law for strong coupling $g>g_{c}$ with a finite and non-zero value of a critical point $g_{c}$. This confinementdeconfinement transition corresponds exactly to the BKT transition.

\section{$3.2 \quad \mathrm{O}(2)$ NLSM and Wilson loop}

Defining the angle variable $\varphi(z)$ for $U(z) \in U(1)$,

$$
U(z)=e^{i \varphi(z)}
$$

we obtain

$$
\omega_{\mu}(z)=\frac{i}{g} U(z) \partial_{\mu} U^{\dagger}(z)=\frac{1}{g} \partial_{\mu} \varphi(z)
$$

Then the action of $\mathrm{O}(2)$ NLSM reads

$$
S_{O(2)}[U]=\int d^{D-2} z \pi \omega_{\mu}(z) \omega_{\mu}(z)=\frac{\beta}{2} \int d^{D-2} z \partial_{\mu} \varphi(z) \partial_{\mu} \varphi(z), \quad \beta:=\frac{2 \pi}{g^{2}} .
$$


The partition function is defined by

$$
Z_{O(2)}[U]=\int[d U] \exp \left(-S_{O(2)}[U]\right), \quad[d U]=\prod_{x} \frac{d \varphi(x)}{2 \pi},
$$

using the Haar measure $d U$ on $\mathrm{U}(1)$. For the notation of the two-dimensional vector,

$$
\mathbf{S}(x)=(\cos \varphi(x), \sin \varphi(x)),
$$

the action reads

$$
S_{O(2)}[U]=\frac{\beta}{2} \int d^{D-2} z \partial_{\mu} \mathbf{S}(x) \cdot \partial_{\mu} \mathbf{S}(x) .
$$

Hence the $\mathrm{O}(2)$ NLSM is regarded as a continuum version of classical planar spin model. In the following, we identify $\beta$ with the inverse temperature $1 / T$. Hence the high (resp. low) temperature of the spin model corresponds to strong (resp. weak) coupling of the gauge theory.

It should be remarked that $\mathrm{O}(2)$ NLSM with the action (3.22) is not a free scalar field theory, since this theory is periodic in the angle variable $\varphi$ (modulo $2 \pi$ ). Of course, if we neglect this periodicity and treat the variable $\varphi$ as a non-compact variable $\varphi(x) \in(-\infty,+\infty)$, we have a trivial theory, i.e., free massless scalar field theory. In this case, the Contour integral is zero,

$$
\oint_{C} \omega_{\mu}(z) d z^{\mu}=\frac{1}{g} \oint_{C} \partial_{\mu} \varphi(z) d z^{\mu}=0
$$

Hence, the Wilson loop $W_{c}[\omega]$ is trivial, $W_{c}[\omega] \equiv 1$ and hence the total static quark potential comes from the the Wilson loop expectation $W_{c}[v]$ of perturbative $\mathrm{U}(1)$ gauge theory and is equal to the Coulomb potential. Thus we obtain a trivial result that the four-dimensional non-compact Abelian gauge theory fails to confine quarks (charges).

However, the periodicity (or compactness) leads to topological non-trivial solutions which are seeds for confinement, as shown in what follows.

In the following, we restrict our consideration to $d:=D-2=2$ case. The extremum of the classical action (3.22) is obtained as a solution of the classical field equation,

$$
\nabla^{2} \varphi=0 \quad(\bmod 2 \pi)
$$

The harmonic function $\varphi$ is constant or has singularities. We require $\varphi$ be constant at infinity and assume only isolated singularities around which $\varphi$ varies by $\pm 2 \pi$ as one turns anticlockwise. Then the solution f is written as

$$
\varphi(z)=\sum_{i} Q_{i} \arctan \frac{\left(z-z_{i}\right)_{2}}{\left(z-z_{i}\right)_{1}}=\sum_{i} Q_{i} \operatorname{Im} \ln \left(z-z_{i}\right), \quad z:=x_{1}+i x_{2} .
$$

\footnotetext{
${ }^{2}$ The two-dimensional Laplace equation is equivalent to the Cauchy-Riemann equation. Hence the solution is given by the holomorphic function. If one avoids branch cuts, it is a meromorphic function.
} 
This denotes a sum of vortex excitations located at points $x_{i} \in R^{2}$ and of vorticity $Q_{i}$ (integers). The solution has an alternative form,

$$
\varphi(z)=\ln \prod_{i} \frac{\left(z-z_{i}^{+}\right) /\left|z-z_{i}^{+}\right|}{\left(z-z_{i}^{-}\right) /\left|z-z_{i}^{-}\right|}=\sum_{i}\left[\ln \frac{\left(z-z_{i}^{+}\right)}{\left|z-z_{i}^{+}\right|}-\ln \frac{\left(z-z_{i}^{-}\right)}{\left|z-z_{i}^{-}\right|}\right] .
$$

This means that vortices of intensity \pm 1 are centered at the points $z_{i}^{ \pm}$and that intensities of higher magnitude are obtained when several $z_{i}^{+}$(or $z_{i}^{-}$) coincide. Note that the angle $\varphi$ is a multi-valued function, but $e^{i \varphi}$ is well-defined everywhere, except at the singular points.

The contribution of the solution (3.28) to $\omega_{\mu}$ is

$$
\omega_{\mu}(z)=\frac{1}{g} \partial_{\mu} \varphi(z)=\frac{1}{g} \sum_{i} Q_{i} \epsilon_{\mu \nu} \frac{\left(z-z_{i}\right)_{\nu}}{\left(z-z_{i}\right)^{2}}=\frac{1}{g} \sum_{i} Q_{i} \epsilon_{\mu \nu} \partial_{\nu} \ln \left|z-z_{i}\right| .
$$

Therefore, the integral of one-form $\omega:=\omega_{\mu} d x^{\mu}$ along the closed loop $C$ is

$$
\oint_{C} \omega_{\mu} d x^{\mu}=\oint_{C} \omega=\frac{1}{g} \sum_{i} \int_{0}^{2 \pi} d \Theta_{i}=\sum_{i} \frac{2 \pi}{g} Q_{i}
$$

where the sum runs over all the vortices inside the closed loop $C$ and $\Theta_{i}$ is an angle around $z=z_{i}$,

$$
\Theta_{i}(z):=\arctan \frac{\left(z-z_{i}\right)_{2}}{\left(z-z_{i}\right)_{1}}
$$

Note that $\omega$ is a closed form, $d \omega=0$, but it is not an exact form, that is, there does not exist a function (zero-form) such that $\omega=d f$ with $f$ being defined everywhere in $R^{2}-\{0\}$. Domain of $f(z)=\Theta(z)$ is restricted to $R^{2}-R_{+}$, in other words, for one unit vortex at the origin,

$$
\omega_{\mu}=\epsilon_{\mu \nu} \frac{x_{\nu}}{x^{2}}-2 \pi \theta\left(x_{1}\right) \delta\left(x_{2}\right) \delta_{\mu 2} .
$$

This is analogous to the case of the magnetic monopole in three dimensions where the magnetic field is given by

$$
H_{\mu}=\frac{1}{2} \frac{x_{\mu}}{|x|^{3}}-2 \pi \delta_{3 \mu} \delta\left(x_{1}\right) \delta\left(x_{2}\right) \theta\left(x_{3}\right) .
$$

The singular line of (3.33) in two dimensions does not contribute to the action, so does the Dirac string (on the positive $Z$ axis) in three dimensions.

In order to calculate the classical action for the singular configuration (3.28), we consider a disk of radius $R_{0}, D_{i}:=\left\{\left|z-z_{i}\right|<R_{0} ; z \in R^{2}\right\}$ (centered on each singular point $z_{i}$ ) which is small with respect to the distances between vortices. Let $\mathcal{R}$ be the remaining domain of integration outside the vortices. The classical action consists of two parts, the self-energy (action) part of vortices,

$$
S^{(1)}=\frac{\beta}{2} \sum_{i} \int_{\left|z-z_{i}\right|<R_{0}} d^{2} z(\nabla \varphi(z))^{2}
$$


and the remaining part,

$$
\begin{aligned}
S^{(2)} & :=\frac{\beta}{2} \int_{\mathcal{R}} d^{2} z(\nabla \varphi(z))^{2} \\
& =-2 \pi \beta \sum_{i \neq j} Q_{i} Q_{j} \ln \left|z_{i}-z_{j}\right|+\sum_{i} Q_{i}^{2} \pi \beta \ln 1 / R_{0} .
\end{aligned}
$$

Summing over all vortex sectors leads to the partition function of the form,

$$
Z_{C}=\sum_{n=0}^{\infty} \frac{\zeta^{n}}{(n !)^{2}} \int \prod_{j=1}^{n} d^{2} z_{j} \exp \left[(2 \pi)^{2} \beta \sum_{i, j} Q_{i} Q_{j} \Delta\left(z_{i}, z_{j}\right)\right], \quad \zeta:=e^{-S^{(1)}},
$$

where $\zeta$ comes from the self-energy (action) part of vortices and $\Delta$ expresses the two-dimensional inverse Laplacian given by

$$
\Delta(x, 0)=\frac{1}{2 \pi} \ln \frac{R}{|x|} .
$$

Therefore the partition function just agrees with the two-dimensional neutral Coulomb gas (i.e., a gas of classical charged particles with a Coulomb interaction and globally neutral, $\left.\sum_{i} Q_{i}=0\right)$.

The transition temperature is estimated as follows. The contribution to the free energy from one vortex pair at distance $r_{12}$ in a box of linear dimension $L$ is

$$
\begin{aligned}
F & \sim \ln \int_{\left|z_{1}-z_{2}\right|>R_{0}} d^{2} z_{1} d^{2} z_{2} \exp \left(-2 \pi \beta \ln \left|z_{1}-z_{2}\right| / R_{0}\right) \\
& \sim \ln \left[L^{4} \exp \left(-2 \pi \beta \ln L / R_{0}\right)\right] \\
& \sim(4-2 \pi \beta) \ln L .
\end{aligned}
$$

The vortices always arise in pairs of opposite Coulomb charges to yield finite energy configurations and each pair forms an elementary dipole (Coulomb dipole gas). If $2 \pi \beta>4$ (the low temperature or weak coupling phase), the contribution from the vortex pair is negligible in the limit $L \rightarrow \infty$. In this phase, charges are bound and one has a dielectric medium. As $\beta \rightarrow \infty$, few vortices are present and their correlation decreases rapidly with the relative distance. This describes a dielectric medium of neutral bound states. In this regime, the correlation $\left\langle U(z) U\left(z^{\prime}\right)\right\rangle$ decays polynomially,

$$
\left\langle U(z) U\left(z^{\prime}\right)\right\rangle=\left|z-z^{\prime}\right|^{-\frac{1}{4 \pi \beta}} .
$$

On the other hand, if $2 \pi \beta<4$ (the high temperature or strong coupling phase), an instability occurs and the creation of well-separated vortices is favored and disorder increases. In high temperature phase, one has a plasma of free charges. The vortex expectation decays exponentially yielding exponential decay of $\left\langle U(z) U\left(z^{\prime}\right)\right\rangle$,

$$
\left\langle U(z) U\left(z^{\prime}\right)\right\rangle=\left|z-z^{\prime}\right|^{-\frac{1}{4 \pi \beta}} e^{-m(\beta)\left|z-z^{\prime}\right|} .
$$

Hence a naive estimate of the critical temperature is obtained

$$
\beta_{c}=\frac{2}{\pi}, \quad g_{c}^{2}=\pi^{2} .
$$


This is the phase transition without the appearance of a spontaneous magnetization. The phase transition can be interpreted as a dipole condensation. The critical point separates the dissociated dipole phase from the condensed phase.

In order to calculate the Wilson loop, we use the equivalence of the Coulomb gas to the sine-Gordon model (see Appendix A for a proof). The sine-Gordon model is defined by the action and the partition function,

$$
\begin{aligned}
S_{s G}(\phi) & :=\alpha \int d^{d} x\left[\frac{1}{2}\left(\partial_{\mu} \phi(x)\right)^{2}-h \cos \phi(x)\right], \\
Z_{s G}(h) & :=\int[d \phi] \exp \left[-S_{s G}(\phi)\right] .
\end{aligned}
$$

This is equivalent to the partition function of a globally neutral gas of particles of charges $Q_{i}= \pm 1$ through a Coulomb potential in $d$ dimensions,

$$
\begin{gathered}
Z_{C, \pm 1}(h)=\sum_{n=0}^{\infty} \frac{z^{2 n}}{(n !)^{2}} \prod_{i=1}^{n} \int d^{d} x_{i} d^{d} y_{j} \exp \left\{-\frac{1}{T}\left[\sum_{i<j}\left[V\left(\left|x_{i}-x_{j}\right|\right)+V\left(\left|y_{i}-y_{j}\right|\right)\right]\right.\right. \\
\left.\left.-\sum_{i, j} V\left(\left|x_{i}-y_{j}\right|\right)\right]\right\}
\end{gathered}
$$

at temperature $T$ with the fugacity $z$,

$$
\alpha=T=\frac{1}{4 \pi^{2} \beta}=\frac{g^{2}}{8 \pi^{3}}, \quad \alpha h=2 z=2 \zeta .
$$

Note that $\alpha \beta=1 /\left(4 \pi^{2}\right)$. It is known that the transition point of sine-Gordon model is

$$
\alpha_{c}=\frac{1}{8 \pi}
$$

which is in agreement with (3.42). The relation of $\varphi$ and $\phi$ is given by

$$
\phi(x, t)=\int_{x}^{\infty} d y \dot{\varphi}(y, t)
$$

or

$$
\partial_{\mu} \varphi=\epsilon_{\mu \nu} \partial^{\nu} \phi
$$

This implies that the fields $\varphi$ and $\phi$ are dual variables. Therefore, O(2) NLSM is equivalent to the Coulomb gas and moreover it is equivalent to the sine-Gordon model when charge of the Coulomb gas (or vorticity of $\mathrm{O}(2)$ NLSM) is restricted to $Q_{i}= \pm 1$. Taking into account $Q_{i}>1$ will lead to the $\cos (Q \phi)$ term. The above consideration can be transferred into the lattice formulation, see [23].

\subsection{Wilson loop and area decay}

The Wilson loop expectation is calculated using the equivalent sine-Gordon model. The generating functional for the charge density $\rho$

$$
\rho(x):=\sum_{i} Q_{i} \delta^{(2)}\left(x-x_{i}\right)
$$


is obtained as

$$
\begin{aligned}
\frac{Z_{s G}[\eta]}{Z_{s G}(0)} & =\left\langle e^{i \int d^{2} x \rho(x) \eta(x)}\right\rangle_{s G}=\left\langle e^{i \sum_{i} Q_{i} \eta\left(x_{i}\right)}\right\rangle_{s G} \\
Z_{s G}[\eta] & =\int[d \phi] \exp \left\{-\alpha \int d^{2} x\left[\frac{1}{2}\left(\partial_{\mu} \phi(x)\right)^{2}-h \cos [\phi(x)+\eta(x)]\right]\right\} .
\end{aligned}
$$

In two dimensions, we can introduce the dual vector field,

$$
H_{\mu}=\epsilon_{\mu \nu} \omega_{\nu}
$$

Then the dual field is connected with the charge density $\rho$ as follows,

$$
\oint_{C} \omega_{\mu}(z) d z^{\mu}=\int_{S} \epsilon_{\mu \nu} \partial_{\mu} \omega_{\nu}(z) d^{2} z=\int \partial_{\mu} H_{\mu}(z) d^{2} z=\frac{2 \pi}{g} \int \rho(z) d^{2} z .
$$

Note that the rotation of $\omega_{\mu}$ or the divergence of $H_{\mu}$

$$
\epsilon_{\mu \nu} \partial_{\mu} \omega_{\nu}=\partial_{\mu} H_{\mu}=\frac{2 \pi}{g} \rho
$$

measures the density of topological charge. If we identify the right-hand-side with the magnetic charge, this implies Dirac quantization condition,

$$
g_{m}=\frac{2 \pi}{g} Q \quad(Q: \text { integer })
$$

The Wilson loop is calculated from the $\eta$ given by

$$
\eta(x)=\frac{q}{g} \oint_{C} d z^{\mu} \epsilon_{\mu \nu} \frac{(z-x)_{\nu}}{(z-x)^{2}}
$$

since

$$
q \oint_{C} \omega_{\mu} d z^{\mu}=\int d^{d} x \rho(x) \eta(x)=\sum_{i} Q_{i} \eta\left(x_{i}\right)
$$

Note that $\eta(x)=0$ if the argument $x$ of $\eta(x)$ is outside the loop, while $\eta(x)=2 \pi q / g$ if $x$ is inside the loop.

In high temperature phase, photon is massive, whereas photon is massless in the low temperature phase. This is because in the high temperature phase, random distribution of free vortices with long range interaction spoil the correlation. Therefore, in the high temperature phase, the Wilson loop expectation is estimated by the steepest descent as

$$
\left\langle W_{C}[\omega]\right\rangle_{s G} \cong \exp \left\{-\alpha \int d^{2} x\left[\frac{1}{2}\left(\partial_{\mu}\left[\phi_{c l}(x)-\eta(x)\right]\right)^{2}-h \cos \phi_{c l}(x)\right]\right\},
$$

where $\phi_{c l}$ is determined by the Debye equation,

$$
\nabla^{2}\left[\phi_{c l}(x)-\eta(x)\right]=h \sin \phi_{c l}(x) .
$$


Corrections to this field due to fluctuation are exponentially small in high temperature phase. The loop $C$ is placed in two-dimensional plane. We can perform the calculation in the same way as done by Polyakov [3].

Instead of repeating similar calculation to [3], we use the Villain form [27],

$$
e^{J \cos \phi} \rightarrow e^{J} \sum_{m \in Z} e^{-\frac{J}{2}(\phi-2 \pi m)^{2}}
$$

to estimate the Wilson loop expectation. Then the partition function is replaced with (apart from field-independent constants)

$$
\begin{aligned}
Z_{s G}[\eta]= & \int[d \phi] e^{-\alpha \int d^{d} x\left[\frac{1}{2}\left(\partial_{\mu} \phi(x)\right)^{2}\right]} \prod_{x \in R^{d}} e^{\alpha h \cos [\phi(x)+\eta(x)]} \\
= & \int[d \phi] e^{-\alpha \int d^{d} x\left[\frac{1}{2}\left(\partial_{\mu} \phi(x)\right)^{2}\right]} \prod_{x \in R^{d}} \sum_{m(x) \in Z} e^{-\frac{\alpha h}{2}[\phi(x)+\eta(x)-2 \pi m(x)]^{2}} \\
= & \sum_{\left\{m(x) \in Z ; x \in R^{d}\right\}} \int[d \phi] e^{-\alpha \int d^{d} x \frac{1}{2}\left[-\phi(x) \partial^{2} \phi(x)\right]} e^{-\frac{\alpha h}{2} \int d^{d} x[\phi(x)+\eta(x)-2 \pi m(x)]^{2}} \\
= & \sum_{\left\{m(x) \in Z ; x \in R^{d}\right\}} e^{-\frac{\alpha h}{2} \int d^{d} x[\eta(x)-2 \pi m(x)]^{2}} \\
& \times \int[d \phi] e^{-\int d^{d} x\left\{\frac{\alpha}{2} \phi(x)\left(-\partial^{2}+h\right) \phi(x)+\alpha h \phi(x)[\eta(x)-2 \pi m(x)]\right\}} \\
= & \sum_{\left\{m(x) \in Z ; x \in R^{d}\right\}} \exp \left[-\frac{\alpha h}{2} \int d^{d} x\left\{[\eta(x)-2 \pi m(x)]^{2}\right.\right. \\
& \left.\left.-h[\eta(x)-2 \pi m(x)]\left(-\partial^{2}+h\right)^{-1}[\eta(x)-2 \pi m(x)]\right\}\right]
\end{aligned}
$$

When $\eta=0$, the denominator is obtained,

$$
Z_{s G}[0]=\sum_{\left\{m(x) \in Z ; x \in R^{d}\right\}} \exp \left[-(2 \pi)^{2} \frac{\alpha h}{2} \int d^{d} x\left\{m(x)^{2}-h m(x)\left(-\partial^{2}+h\right)^{-1} m(x)\right\}\right](3
$$

Note that the field $\eta(x)$ has its support on $S(\partial S=C)$ and has the value $2 \pi(q / g)$. If $q$ is an integral multiple of $g$ (the elementary charge), we have $\eta \in 2 \pi Z$. This is absorbed by the shift of $m$. Therefore, in this case, charge confinement does not occur. This is interpreted as the charge screening.

A naive estimate of the ratio $Z_{s G}[\eta] / Z_{s G}[0]$ is given when $\eta \notin 2 \pi Z$ in Appendix B. Finally we obtain the area decay of the Wilson loop expectation,

$$
\begin{aligned}
\left\langle W_{C}[\omega]\right\rangle_{s G} & \cong e^{-\sigma A(C)} \\
\sigma & =\left(2 \pi \frac{q}{g}\right)^{2} \frac{\alpha h}{2}=\left(2 \pi \frac{q}{g}\right)^{2} \zeta \sim e^{-S^{(1)}} .
\end{aligned}
$$

This implies the linear static potential

$$
V(R)=\sigma R
$$




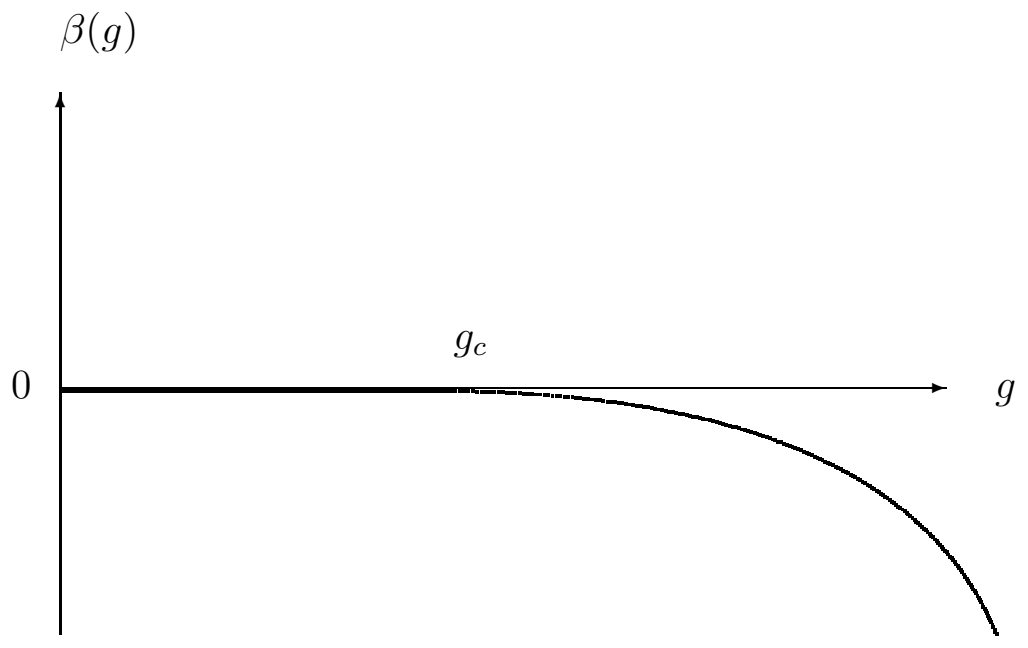

Figure 1: Renormalization group beta function of U(1) gauge theory

between two fixed electric charges and electric string with uniform energy density $\sigma$ which is called the string tension. Therefore condensation of topological non-trivial configuration leads to quark confinement. From (3.17), (3.19) and (3.66), the total static potential is given by

$$
V(R)=\sigma R-\frac{g^{2}}{4 \pi} \frac{1}{R}+\text { constant. }
$$

Even the continuum Abelian U(1) gauge theory has a confinement phase, exhibiting rich phase structure as in lattice compact $\mathrm{U}(1)$ gauge theory.

\section{Discussion}

In the four-dimensional pure $\mathrm{U}(1)$ gauge theory, we have proved the existence of a strong coupling phase where the fractional electric charge is confined by the linear static potential due to vortex condensation. In the following we discuss a few points of perspective.

\subsection{Renormalization group and non-Gaussian fixed point}

The $\mathrm{O}(\mathrm{N})$ NLSM has the renormalization group beta function,

$$
\beta(g):=\mu \frac{d g(\mu)}{d \mu}=-\frac{N-2}{8 \pi^{2}} g^{3}+O\left(g^{5}\right) .
$$

In the low temperature $\left(0<T<T_{c}\right)$, therefore, the $\mathrm{O}(2)$ NLSM has vanishing beta function, $\beta \equiv 0 \quad(T \ll 1)$, and $0<T<T_{c}$ is the line of fixed points. This is consistent with the fact that at low temperature, the inverse correlation length or mass $m=\xi^{-1}$ of $\mathrm{O}(2) \mathrm{NLSM}$ or XY model vanishes, i.e., $m(T) \equiv 0$ for all $T<T_{c}$. The 
theory is conformal invariant. For high temperature $\left(T>T_{c}\right)$, the renormalization group study of XY model shows that the mass behaves as

$$
m(T) \sim \exp \left(-\frac{C}{\sqrt{T-T_{c}}}\right), \quad T \downarrow T_{c},
$$

with a constant $C$.

These results established in two-dimensional model would be translated into the four-dimensional Abelian gauge theory, provided that two theories have the same renormalization-group beta function. We assume that in two theories the mass $m(g)$ is generated by the dimensional transmutation in such a way that the beta function $\beta(g)$ is related to the mass $m(g)$ through the well-known relation,

$$
m(g)=\mu f(g)=\mu \exp \left(-\int^{g} \frac{d g}{\beta(g)}\right),
$$

where $\beta(g)$ is defined by

$$
\beta(g):=\mu \frac{d g(\mu)}{d \mu}=-\frac{f(g)}{f^{\prime}(g)} .
$$

In the weak coupling phase $\left(g<g_{c}\right)$, the gauge field is massless, $m(g) \equiv 0$ and the beta function of the renormalization group [22] is identically zero,

$$
\beta(g) \equiv 0 \quad\left(0<g<g_{c}\right) .
$$

Therefore, $0<g<g_{c}$ is the line of fixed points. In strong coupling phase $\left(g>g_{c}\right)$, the beta function behaves as (see Figure 1)

$$
\beta(g)=-\frac{1}{B g_{c}}\left(g^{2}-g_{c}^{2}\right)^{3 / 2}<0, \quad\left(g>g_{c}, g \cong g_{c}\right),
$$

which is compatible with the BKT mass (4.69),

$$
m(g)=A \exp \left(-\frac{B}{\sqrt{g^{2}-g_{c}^{2}}}\right), \quad g \downarrow g_{c},
$$

with constants $A, B>0$. Note that $\beta(g)$ is independent of $A$.

It is worth remarking that the recent lattice computer simulation [28, 29] indicates the existence of an non-Gaussian fixed point in four-dimensional pure compact $\mathrm{U}(1)$ gauge theory. This should be compared with the old results 30, 31. In the simulation [28, 29] the continuous phase transition was found and analyzed according to the power law scaling, although our investigation suggests the scaling behavior of the essential singularity type and the data do not exclude the essential singularity. It will be rather difficult to specify the essential singularity in the computer simulations, since the lattice size available is not yet so large to confirm this issue. Anyway, it will be interesting to find any relationship to fill the gap between two approaches. 
If the mass scale is generated by dimensional transmutation, the above results are quite analogous to the situation found for the dynamical fermion mass and the beta function in quenched massless QED [32, 33]. The relationship of these results with the quenched QED is more suggestive by the method of bosonization or fermionization. The two-dimensional sine-Gordon model is equivalent to the massive Thirring model 34, 35 with an action,

$$
S[\psi, \bar{\psi}]=\int d^{2} x\left[\bar{\psi}\left(i \gamma_{\mu} \partial_{\mu}+m\right) \psi-\frac{G}{2}\left(\bar{\psi} \gamma_{\mu} \psi\right)^{2}\right]
$$

The correspondence between two theories is given by

$$
\begin{aligned}
1+\frac{G}{\pi} & =4 \pi \alpha \\
\bar{\psi} \gamma_{\mu} \psi & =-\frac{1}{2 \pi} \epsilon_{\mu \nu} \partial_{\nu} \phi \\
m \bar{\psi} \psi & \rightarrow-\alpha h \cos \phi .
\end{aligned}
$$

Our result shows that the transition point $\alpha_{c}=1 /(8 \pi)$ corresponds to $G_{c}=-\pi / 2$. By making use of this equivalence, the Wilson loop (3.64) in the topological nontrivial sector of the four-dimensional $\mathrm{U}(1)$ gauge theory can be calculated in the two-dimensional massive Thirring model. The details will be given elsewhere.

\subsection{Lower and higher dimensional cases}

Using the equivalence between the TQFT part of $\mathrm{U}(1)_{D}$ gauge theory and $\mathrm{O}(2)_{D-2}$ NLSM, we can study other dimensional cases.

For $D=3$, the equivalent $\mathrm{O}(2)$ NLSM is one dimensional, $\mathrm{O}(2)_{1}$. This is not a field theory model, but a quantum mechanical model of the plane rotor. There is no phase transition in this model. This implies that the three-dimensional U(1) gauge theory has only the confinement phase. This will be understood as the tunneling effect among classical vacua in the sense that the double-well anharmonic oscillator is related to the one-dimensional Ising model. It will be interesting to see agreement (or disagreement) on confinement mechanism between our approach and the Polyakov approach [2, 3].

For $D=5$, the $\mathrm{O}(2)$ NLSM is three dimensional, $\mathrm{O}(2)_{3}$. The three-dimensional $\mathrm{O}(2)$ NLSM has two phases on the lattice [36]. The phase transition is first order. This result is consistent with the mean field study of five-dimensional lattice $\mathrm{U}(1)$ gauge theory [24]. This theory has a finite non-zero critical coupling $g_{c}$. In the strong coupling phase, quark confinement is expected to occur. However, the phase transition is first order. Therefore, on the lattice, it is impossible to take the continuum limit at this point. In view of this, the construction of continuum $U(1)$ gauge theory from the lattice regularized theory is problematic in five dimensions and higher dimensions, unless the action is modified. 


\section{Acknowledgment}

The author would like to thank Jiri Jersak and Volodya Miransky for informing him of the recent results of the computer simulation [28, 29]. This work is supported in part by the Grant-in-Aid for Scientific Research from the Ministry of Education, Science and Culture.

\section{A Equivalence between Coulomb gas and sine-Gordon model}

The partition function can be rewritten as

$$
\begin{aligned}
Z_{s G}(h) & =\int[d \phi] e^{-\alpha \int d^{d} x \frac{1}{2}\left(\partial_{\mu} \phi(x)\right)^{2}} \sum_{n=0}^{\infty} \frac{(\alpha h)^{n}}{n !}\left[\int d^{d} x \cos \phi(x)\right]^{n} \\
& =\int[d \phi] e^{-\alpha \int d^{d} x \frac{1}{2}\left(\partial_{\mu} \phi(x)\right)^{2}} \sum_{n=0}^{\infty} \frac{(\alpha h)^{2 n}}{(2 n) !} \frac{1}{2^{2 n}}\left[\int d^{d} x\left(e^{i \phi(x)}+e^{-i \phi(x)}\right)\right]^{2 n} \\
& =\int[d \phi] e^{-\alpha \int d^{d} x \frac{1}{2}\left(\partial_{\mu} \phi(x)\right)^{2}} \sum_{n=0}^{\infty} \frac{(\alpha h)^{2 n}}{(2 n) !} \frac{1}{2^{2 n}}\left(\begin{array}{c}
2 n \\
n
\end{array}\right) \prod_{i=1}^{n} \int d^{d} x_{i} d^{d} y_{i} e^{i \phi\left(x_{i}\right)-i \phi\left(y_{i}\right)} \\
& =\int[d \phi] e^{-\alpha \int d^{d} x \frac{1}{2}\left(\partial_{\mu} \phi(x)\right)^{2}} \sum_{n=0}^{\infty} \frac{(\alpha h / 2)^{2 n}}{(n !)^{2}} \prod_{i=1}^{n} \int d^{d} x_{i} d^{d} y_{i} e^{i \phi\left(x_{i}\right)-i \phi\left(y_{i}\right)}
\end{aligned}
$$

Note that

$$
\begin{aligned}
{\left[\left[e^{\int d^{d} x J(x) \phi(x)}\right]\right] } & :=\int[d \phi] \exp \left[-\frac{\alpha}{2} \int d^{d} x\left(\partial_{\mu} \phi(x)\right)^{2}+\int d^{d} x J(x) \phi(x)\right] \\
& =\exp \left[\frac{1}{2 \alpha} \int d^{d} x \int d^{d} y J(x) \Delta(x, y) J(y)\right],
\end{aligned}
$$

where $\Delta(x, y)$ is the massless scalar field propagator,

$$
\Delta(x, y):=\int \frac{d^{d} p}{(2 \pi)^{d}} \frac{e^{i p(x-y)}}{p^{2}} .
$$

In particular, for $J(x)=i \sum_{j} q_{j} \delta\left(x-x_{j}\right)$,

$$
\left[\left[\prod_{j=1}^{n} e^{i q_{j} \phi\left(x_{j}\right)}\right]\right]=\left\{\begin{array}{ll}
\exp \left[-\frac{1}{2 \alpha} \sum_{j, k} q_{j} q_{k} \Delta\left(x_{j}, x_{k}\right)\right] & \text { for } \sum_{j} q_{j}=0 \\
0 & \text { for } \sum_{j} q_{j} \neq 0
\end{array},\right.
$$

where the latter case is a result of invariance under constant translation of the field $\varphi(z) \rightarrow \varphi(x)+c$. Using this result for $q_{j}= \pm 1$,

$$
\begin{aligned}
Z_{s G}(h)= & \sum_{n=0}^{\infty} \frac{(\alpha h / 2)^{2 n}}{(n !)^{2}} \prod_{i=1}^{n} \int d^{d} x_{i} d^{d} y_{i} \int[d \phi] e^{-\alpha \int d^{d} x \frac{1}{2}\left(\partial_{\mu} \phi(x)\right)^{2}} \prod_{i=1}^{n} e^{i \phi\left(x_{i}\right)-i \phi\left(y_{i}\right)} \\
= & \sum_{n=0}^{\infty} \frac{(\alpha h / 2)^{2 n}}{(n !)^{2}} \prod_{i=1}^{n} \int d^{d} x_{i} d^{d} y_{i} \exp \left\{-\frac{1}{2 \alpha}\left[\sum_{i<j}\left[\Delta\left(x_{i}-x_{j}\right)+\Delta\left(y_{i}-y_{j}\right)\right]\right.\right. \\
& \left.\left.\quad-\sum_{i, j} \Delta\left(x_{i}-y_{j}\right)\right]\right\}
\end{aligned}
$$




\section{B Estimate of sine-Gordon partition function}

Note that the quantity

$$
\varrho(x)^{2}-h \varrho(x)\left(-\partial^{2}+h\right)^{-1} \varrho(x)=\varrho(x) \frac{-\partial^{2}}{-\partial^{2}+h} \varrho(x)
$$

is positive, since $\left(-\partial^{2}\right) /\left(-\partial^{2}+h\right)$ is a positive operator. Therefore,

$$
e^{-\frac{\alpha h}{2}(2 \pi)^{2} \int_{S} d^{d} x\left\{\varrho(x)^{2}-h \varrho(x)\left(-\partial^{2}+h\right)^{-1} \varrho(x)\right\}}, \quad \varrho(x)=\left|\frac{\eta(x)}{2 \pi}-m(x)\right|
$$

is monotonically (rapidly) decreasing in $\{|\rho(x)| ; x \in S\}$. Therefore, in the partition function,

$$
Z_{s G}[\eta]=\sum_{\left\{m(x) \in Z ; x \in R^{d}\right\}} \exp \left[-\frac{\alpha h}{2}(2 \pi)^{2} \int d^{d} x\left\{\varrho(x)^{2}-h \varrho(x)\left(-\partial^{2}+h\right)^{-1} \varrho(x)\right\}\right],
$$

the most dominant contribution comes from a set of configurations $\{|\varrho(x)| ; x \in S\}$ which gives the smallest value for $\int d^{d} x\left\{\varrho(x)^{2}-h \varrho(x)\left(-\partial^{2}+h\right)^{-1} \varrho(x)\right\}$.

If the argument $x$ of $\eta(x)$ is outside the loop $C(C=\partial S), \eta(x)=0$, while $\eta(x)=$ $2 \pi q / g$ if $x$ is inside the loop. For $Z_{s G}[0],\{m(x) \equiv 0\}$ gives the most dominant contribution. Hence, we see

$$
Z_{s G}[0]=1+\cdots
$$

For $Z_{s G}[\eta]$, the most dominant contribution comes from a set of integers $\{m(x)\}$ whose value is the nearest to $q / g$ where $\varrho(x)=2 \pi|q / g-m(x)|$. Since the integral part of $q / g$ is absorbed in the shift of $m(x)$, it is sufficient to consider the case $0<q / g<1$ without loss of generality. For a half-integer $q / g$, i.e., $q / g= \pm 1 / 2$, we see that $\{m(x) \equiv 0, \pm 1\}$ give the smallest value of $2 \pi|q / g-m(x)|$ for $x \in S$. For $0<q / g<1 / 2$ (resp. $1 / 2<q / g<1$ ), the most dominant contribution is given by $\{m(x) \equiv 0\}$ (resp. $\{m(x) \equiv 1\}$ ). Thus, the rough estimate, for example, in the case of $0<q / g<1 / 2$ leads to

$$
Z_{s G}[\eta]=e^{-\frac{\alpha h}{2} \int_{S} d^{d} x\left\{\eta(x)^{2}-h \eta(x)\left(-\partial^{2}+h\right)^{-1} \eta(x)\right\}}+\cdots .
$$

A naive estimate of the ratio $Z_{s G}[\eta] / Z_{s G}[0]$ is given for $\eta \notin 2 \pi Z$ by

$$
\left\langle W_{C}[\omega]\right\rangle_{s G}=\frac{Z_{s G}[\eta]}{Z_{s G}[0]}=e^{-\frac{\alpha h}{2} \int_{S} d^{d} x \eta(x)\left[1-h\left(-\partial^{2}+h\right)^{-1}\right] \eta(x)}+\cdots .
$$

Here $\left(-\partial^{2}+h\right)^{-1}(x, y)$ is the massive scalar propagator with mass $m \sim \sqrt{h}$ and hence has an exponential damping factor $e^{-m|x-y|}$. Therefore, the integral $\int_{S} d^{d} x \eta(x)\left(-\partial^{2}+\right.$ $h)^{-1} \eta(x)$ converges to a finite value even for large $S$. The term $\int_{S} d^{2} x \eta(x)^{2}$ is proportional to the area of $S$. This term leads to the area decay of the Wilson loop expectation. 


\section{References}

[1] A.M. Polyakov, Gauge fields and strings (Harwood Academic Publishers, London, 1987).

[2] A.M. Polyakov, Compact gauge fields and the infrared catastrophe, Phys. Lett. B 59, 82-84 (1975).

[3] A.M. Polyakov, Quark confinement and topology of gauge theories, Nucl. Phys. B 120, 429-458 (1977).

[4] K.-I. Kondo, Yang-Mills Theory as a Deformation of Topological Field Theory, Dimensional Reduction and Quark Confinement, hep-th/9801024.

[5] K. Wilson, Confinement of quarks, Phys. Rev. D 10, 2445-2459 (1974).

[6] G. Parisi and N. Sourlas, Random magnetic fields, supersymmetry, and negative dimensions, Phys. Rev. Lett. 43, 744-745 (1979).

[7] N.D. Mermin and H. Wagner, Absence of ferromagnetism or antiferromagnetism in one- or two-dimensional isotropic Heisenberg models, Phys. Rev. Lett. 17, 1133-1136 (1966).

N.D. Mermin, Absence of ordering in certain classical systems, J. Math. Phys. 8, 1061-1064 (1967).

P.C. Hohenberg, Existence of long-range order in one and two dimensions, Phys. Rev. 158, 383-386 (1967).

S. Coleman, There are no Goldstone bosons in two dimensions, Commun. Math. Phys. 31, 259-264 (1973).

[8] V.L. Berezinskii, Destruction of long-range order in one-dimensional and twodimensional systems having a continuous symmetry group I. classical systems, Soviet Physics JETP 32, 493-500 (1971).

J.M. Kosterlitz, The critical properties of the two dimensional XY model, J. Phys. C 7, 1046-1060 (1974).

J.M. Kosterlitz and D.V. Thouless, Ordering, metastability and phase transitions in two-dimensional systems, J. Phys. C 6, 1181-1203 (1973).

[9] J. Fröhlich, Classical and quantum statistical mechanics in one and two dimensions: two-component Yukawa- and Coulomb systems, Commun. Math. Phys. 47, 233-268 (1976).

[10] J.V. Jose, L.P. Kadanoff, S. Kirkpatrick and D.R. Nelson, Renormalization, vortices, and symmetry-breaking perturbations in the two-dimensional planar model, Phys. Rev. B 16, 1217-1241 (1977).

[11] D.J. Amit, Y.Y. Goldschmidt and G. Grinstein, Renormalization group analysis of the phase transition in the 2D Coulomb gas, sine-Gordon theory and XYmodel, J. Phys. A 13, 585-620 (1980).

[12] J. Fröhlich and T. Spencer, The Kosterlitz-Thouless transition in two dimensional abelian spin systems and the Coulomb gas, Commun. Math. Phys. 81, 527-602 (1981).

[13] T. Banks, R. Myerson and J. Kogut, Phase transitions in abelian lattice gauge theories, Nucl. Phys. B 129, 493-510 (1977). 
[14] J. Glimm and A. Jaffe, Instantons in a U(1) lattice gauge theory, Commun. Math. Phys. 56, 195-212 (1977).

[15] M.E. Peskin, Mandelstam-'t Hooft duality in abelian lattice models, Ann. Phys. 113, 122-152 (1978).

[16] K.-I. Kondo, Abelian-projected effective gauge theory of QCD with asymptotic freedom and quark confinement, hep-th/9709109 (revised), Phys. Rev. D 57, No. 12 (1998), in press.

[17] K. Osterwalder and E. Seiler, Gauge field theories on the lattice, Ann. Phys. 110, 440-471 (1978).

[18] S. Elitzur, R.B. Pearson and J. Shigemitsu, Phase structure of discrete Abelian spin and gauge systems, Phys. Rev. D 19, 3698-3714 (1979).

[19] A. Ukawa, P. Windey and A. Guth, Dual variables for lattice gauge theories and phase structure of Z(N) systems, Phys. Rev. D 21, 1013-1036 (1980).

[20] A. Guth, Existence proof of a nonconfining phase in four-dimensional U(1) lattice gauge theory, Phys. Rev. D 21, 2291-2307 (1980).

[21] J. Fröhlich and T. Spencer, Massless phases and symmetry restoration in Abelian gauge theories and spin systems, Commun. Math. Phys. 83, 411-454 (1982).

[22] K.G. Wilson and J. Kogut, The renormalization group and the $\epsilon$ expansion, Phys. Rep. 12 C, 75-200 (1974).

[23] J.B. Kogut, Introduction to lattice gauge theory and spin systems, Rev. Mod. Phys. 51, 659-713 (1979).

[24] J.-M. Drouffe and J.-B. Zuber, Strong coupling and mean field methods in lattice gauge theories, Phys. Rep. 102, 1-119 (1983).

[25] E. Seiler, Gauge theories as a problem of constructive field theory and statistical mechanics, Lecture Note in Physics, Vol. 159 (Springer, 1982).

[26] K.-I. Kondo, Abelian magnetic monopole dominance in quark confinement, hepth/9805153.

[27] J. Villain, Theory of one- and two-dimensional magnets with an easy magnetization plane. II. The planar, classical, two-dimensional magnet, J. Physique 36, 581-590 (1975).

[28] J. Jersak, C.B. Lang and T. Neuhaus, Non-Gaussian fixed point in fourdimensional pure compact $\mathrm{U}(1)$ gauge theory on the lattice, hep-lat/9606010. Phys. Rev. Lett. 77, 1933-1936 (1996).

[29] J. Cox, W. Franzki, J. Jersak, C.B. Lang, T. Neuhaus and P.W. Stephenson, Gauge-ball spectrum of the four-dimensional pure U(1) gauge theory, heplat/9701005, Nucl. Phys. B 499, 371-408 (1997). 
[30] H.G. Evertz, J. Jersak, T. Neuhaus and P.M. Zerwas, Tricritical point in lattice QED, Nucl. Phys. B 251 [FS13], 279-298 (1985).

[31] J. Jersak, T. Neuhaus and P.M. Zerwas, Charge renormalization in compact lattice QED, Nucl. Phys. B 251 [FS13], 299-310 (1985).

[32] V.A. Miransky, Dynamics of spontaneous chiral symmetry breaking and the continuum limit in quantum electrodynamics, Nuovo Cimento 90A, 149-170 (1985).

[33] K.-I. Kondo, T. Mino and K. Yamawaki, Critical line and dilaton in scaleinvariant QED, Phys. Rev. D 39, 2430-2433 (1989).

[34] S. Coleman, Quantum sine-Gordon equation as the massive Thirring model, Phys. Rev. D 11, 2088-2097 (1975).

[35] K.-I. Kondo, Bosonization and duality of massive Thirring model, Prog. Theor. Phys. 94, 899-914 (1995).

[36] P.R. Thomas and M. Stone, Nature of the phase transition in a non-linear $O(2)_{3}$ model, Nucl. Phys. B 144, 513-524 (1978). 